\title{
Water enema computed tomography (WE-CT) in the local staging of low colorectal neoplasms: comparison with transrectal ultrasound
}

\author{
F. Caseiro-Alves, ${ }^{1}$ M. Gonçalo, ${ }^{1}$ L. Cruz, ${ }^{1}$ J. Ilharco,,${ }^{1}$ J. Leite, ${ }^{2}$ A. Agostinho, ${ }^{1}$ F. Castro e Sousa, ${ }^{2}$ \\ H. Vilaça-Ramos ${ }^{1}$ \\ ${ }^{1}$ Serviço de Radiologia, Hospital Universidade de Coimbra, Praceta Mota Pinto, 3000-Coimbra, Portugal \\ ${ }^{2}$ Serviço de Cirurgia, Hospital Universidade de Coimbra, Praceta Mota Pinto, 3000-Coimbra, Portugal
}

Received: 30 January 1997/Accepted: 5 March 1997

\begin{abstract}
Background: To determine the accuracy of computed tomography performed with a water enema application (WE-CT) in the local staging of low colorectal neoplasms and to compare the results with those of transrectal ultrasonography (TRUS).

Methods: Forty patients with low colorectal tumors were evaluated prospectively by $\mathrm{CT}$ with the simultaneous administration of a lukewarm rectal enema (0.5$1.5 \mathrm{~L})$. Thin slices $(5 \mathrm{~mm})$ and intravenous application of iodinated contrast media were routinely used. TRUS was performed in 18 patients. Tumor size, location, and staging according to the TNM classification of the UICC were registered. Tumors were classified as $\angle \mathrm{T} 3$ (T1 or $\mathrm{T} 2$ ) or as T3 or T4. For staging peritumoral lymph node metastases on WE-CT, two criteria of positivity were tested: $\mathrm{N}+$ if at least one peritumoral node $\geq 5 \mathrm{~mm}$ in diameter was seen (reading A); $\mathrm{N}+$ if at least one peritumoral node $\geq 5 \mathrm{~mm}$ or three peritumoral nodes $<5$ $\mathrm{mm}$ were identified (reading B).

Results: For the tumor staging, WE-CT showed a sensitivity of $90 \%$, a specificity of $73 \%$, a positive predictive value (PPV) of $90 \%$, a negative predictive value (NPV) of $73 \%$, and an accuracy of $85 \%$. For TRUS, the results were sensitivity of $73 \%$, specificity of $29 \%$, PPV of $62 \%$, NPV of $40 \%$, and an accuracy of $39 \%$. Concerning nodal staging with WE-CT, results were superior when reading A was used: sensitivity $=84 \%$, specificity $=83 \%, \mathrm{PPV}=73 \%$, NPV $=91 \%$, and accuracy $=84 \%$. TRUS showed a sensitivity of $29 \%$, specificity of $100 \%$, PPV of $100 \%$, NPV of $67 \%$, and an accuracy of $71 \%$.
\end{abstract}

Correspondence to: F. Caseiro-Alves
Conclusion: WE-CT is a reliable technique for the local staging of low colorectal tumors that can be superior to TRUS. For diagnosis of peritumoral metastatic lymph nodes on WE-CT, the 5-mm diameter cutoff value is the most appropriate size criterion.

Key words: Computed tomography - Transrectal ultrasound-Rectal tumors - Preoperative staging.

Optimal management of rectal tumors requires accurate preoperative staging to select properly patients who may benefit from less aggressive surgery or adjuvant therapy [1]. Due to inaccurate staging by clinical examination, several imaging methods have been proposed that use computed tomography (CT), transrectal ultrasonography (TRUS), or, more recently, magnetic resonance imaging (MRI) with transrectal coils. Despite several articles reporting TRUS as the most accurate imaging method for the local staging of rectal tumors [2-4], a recent article [5] reported a trend toward overstaging the degree of tumor penetration within the bowel wall. The reported accuracy of CT has been variable, depending on patient selection (early or advanced neoplasia) and, most importantly, on the technical conditions of the examination (presence or absence of rectal distention) [6-11]. The purpose of our study was twofold: to compare the results of CT performed with a simultaneous water enema technique (WE-CT) with those of TRUS for the local staging of low colorectal tumors and to define which criteria is more useful in CT detection of metastatic local lymph node involvement.

\section{Material and methods}

Forty-seven patients with low colorectal tumors, diagnosed by endoscopy or barium enema, were consecutively enrolled in this study in a 

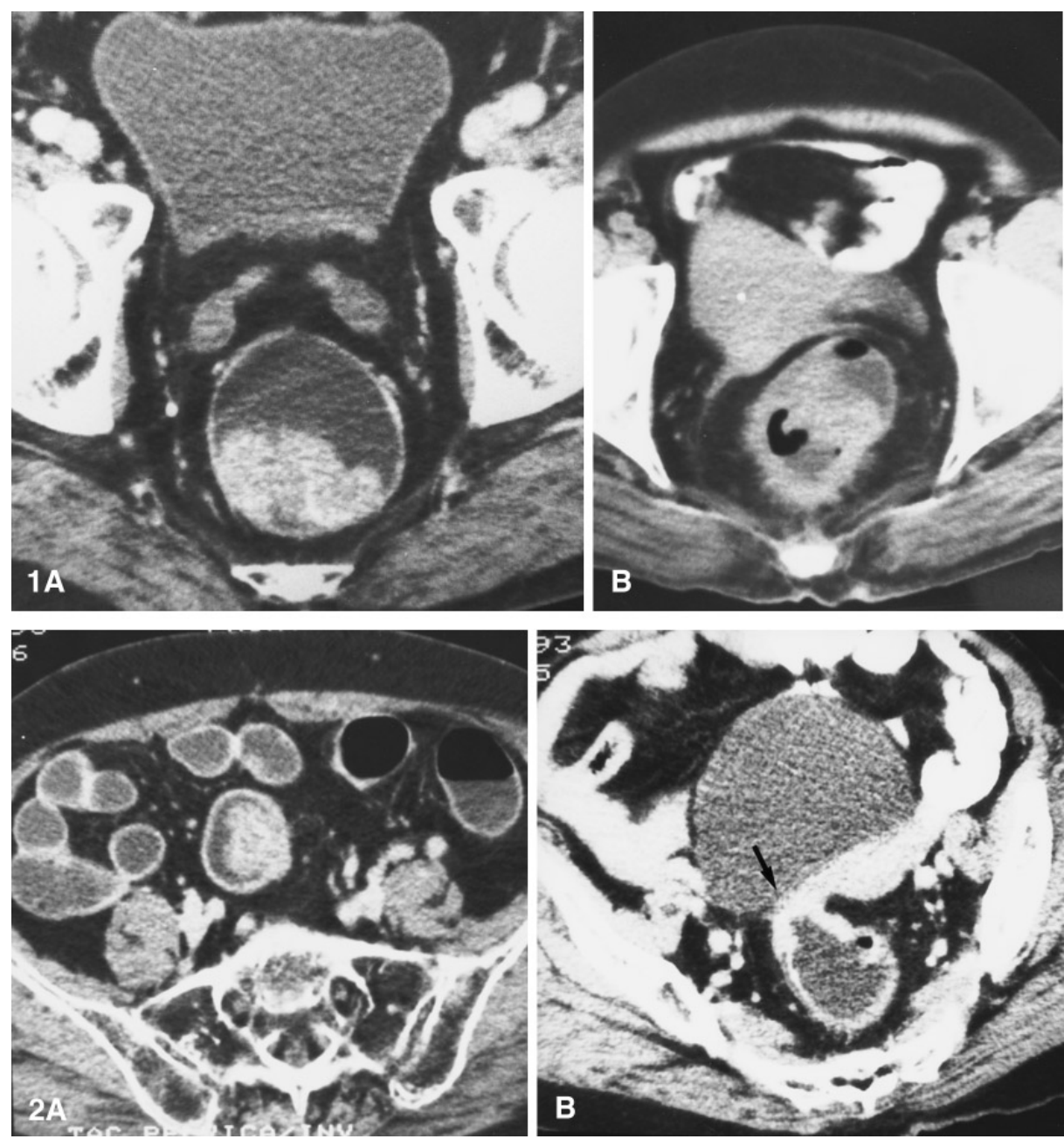

Fig. 1. Imaging classification of the T stage on WE-CT. A Rectal tumor correctly classified as $<\mathrm{T} 3$. Image shows an intraluminal tumor mass without abnormalities of the surrounding fatty tissue. B Rectal adenocarcinoma correctly classified as T3. Note tumor extension to adjacent fat with a spicular appearance.

Fig. 2. False-positive findings of WE-CT. A T2 tumor of the sigmoid colon, located on the left wall. The local bulging of this wall was responsible for incorrect staging as T3. B T3 tumor misdiagnosed as T4 due to presumed invasion of the bladder base (arrow). prospective fashion. Seven patients were excluded due to Crohn disease, with rectal involvement in one case, rectal invasion by prostatic carcinoma in another, surgery performed outside our institution in two, previous pelvic radiotherapy in two, and unavailable pathologic correlation in one. Of the remaining 40 patients, there were 26 man $(65 \%)$ and 14 women $(35 \%)$, with a mean age of 70 years (range $=$ 47-86 years). All of these patients underwent surgery with a mean interval to imaging procedures (CT or TRUS) of 14 days (range $=1-$ 30 days). In 37 patients, anterior resection of the rectum $(n=28)$ or abdominoperineal resection $(n=9)$ was performed. At surgery, three patients had unresectable tumors, and they were surgically staged as T4 Nx.

Patients underwent the same CT protocol, which included small bowel opacification by oral administration of iodinated contrast media and the intramuscular injection of $1 \mathrm{mg}$ of Glucagon (Novo) at the time of examination. Subsequently, $500-1500 \mathrm{~mL}$ of a lukewarm water enema was given and immediately interrupted if the patient experienced discomfort or incontinence. Acceptance of the enema by the patient, volume of instilled water, and the degree of bowel distention were registered. Previous colon cleansing was not used.

Images were performed on a Somatom DR (Siemens, Erlangen, Germany) ( $\mathrm{n}=38$ ) or Somatom ART (Siemens) $(\mathrm{n}=2)$, with a scan time of 4 and $3 \mathrm{~s}$, respectively. Without any gantry tilt, axial 4-mm slices with an interval of $1 \mathrm{~mm}$ were obtained during the intravenous injection of $120 \mathrm{cc}$ of iodinated contrast media at a concentration of $300 \mathrm{mg} \mathrm{I} / \mathrm{mL}$ from the sigmoid level, below the aortic bifurcation, to the anorectum. Tumor size (measured in its greatest diameter), location, and staging according to the TNM classification of the UICC were registered [12]. Images were analyzed by consensus by two readers with previous experience with the technique (F.C.-A., M.G.) blinded to the histological type of the tumor or to results of any other staging procedure. A bowel wall thickening greater than 3-mm was considered abnormal and involved by tumor. Tumors confined to the bowel wall were classified as $<\mathrm{T} 3$ (T1 or T2) (Fig. 1). At the tumor level, any strand of tissue on adjacent fat, a spicular, or irregular appearance of the outer bowel wall contour were considered evidence of perirectal invasion and the tumor classified as T3 (Fig. 1). Loss of intervening fat planes or clear infiltration of adjacent organs was considered evidence of a $\mathrm{T} 4$ stage tumor.

For detection of peritumoral nodal involvement $(\mathrm{N})$ on WE-CT, two different criteria were used to test their individual accuracy. Readings were made randomly on separate sessions. $\mathrm{N}$ was considered positive if at least one peritumoral nodule $\geq 5 \mathrm{~mm}$ in diameter (reading $\mathrm{A}$ ) or at least one peritumoral nodule $\geq 5 \mathrm{~mm}$ in diameter or three peritumoral nodules of any size (reading B) were found.

TRUS, performed in the same day as CT, was attempted in 25 patients. Two other radiologists (L.C., J.I.), blinded to the results of other imaging methods or staging procedures, performed the examinations by using a biplanar electronic transrectal probe with an insonating frequency of 5-7.5 MHz (Endo P, Siemens). Tumors were classified as $>\mathrm{T} 3$ when tumoral penetration of the outer hypoechoic layer corresponding to the muscularis propria was seen. Lymph node in- 
volvement was considered to be present if any solid nodule (irrespective of its size) could be identified on the adjacent perirectal fat.

The standard of reference was the pathological examination of resected specimens, with the register of the total number and size of metastatic lymph nodes. Because unresectable cases precluded any comparison regarding lymph node involvement, they were discarded for the evaluation of this item.

Staging was expressed according to the TNM classification of the UICC [12]. For comparison of tumor size between WE-CT and pathological specimen, Student's t test was performed. Diagnostic accuracy of WE-CT was calculated with an $80 \%$ confidence level.

\section{Results}

At surgery, three tumors were found in the sigmoid colon, nine at the rectosigmoid junction, 23 at the upper two-thirds of the rectum, and five in the lower rectum extending to the anus. Tumors size were $1.5-10 \mathrm{~cm}$ $($ mean $=4.9 \mathrm{~cm})$. Pathological examination revealed adenocarcinoma in 31 cases (27 well differentiated and four moderately differentiated), villous adenoma in eight (six malignant), and basal anal carcinoma in one. Eleven tumors were confined to the bowel wall (T1 = 3 and $\mathrm{T} 2=8$ ), and 24 corresponded to stage $\mathrm{T} 3$ and five to stage $\mathrm{T} 4$.

WE-CT was performed successfully in all cases, but patients with highly stenotic tumors $(n=7)$ did not allow the administration of water volumes greater than $500 \mathrm{~mL}$ due to intolerance or incontinence. Bowel distention was considered satisfactory in all patients except in those cases in which tumor extended low into the anal canal $(n=5)$, where distention was very poor and considered inadequate.

\section{WE-CT findings: staging the tumor}

All tumors were identified at WE-CT. The 31 cases in which pathological record of tumor size was available showed a good correlation with the reported CT size and pathological examination $(p<0.01)$. The tumor was slightly smaller on CT in 17 cases, with a maximal difference of $2 \mathrm{~cm}$ observed in only three cases.

On WE-CT, eight tumors were correctly classified as confined to the bowel wall $(<\mathrm{T} 3)$ and 26 as spreading beyond the wall (T3 or T4), determining a sensitivity of $90 \%$, specificity of $73 \%$, positive predictive value of $90 \%$, negative predictive value of $73 \%$, and an accuracy of $85 \%$ (confidence interval $=0.84-1.95)$ (Table 1$)$. Three cases were overstaged as T3 by WE-CT (Fig. 2). Two of these showed an inflammatory reaction adjacent to the tumor on pathological examination. Three tumors were understaged by WE-CT as $<\mathrm{T} 3$. One of these tumors had a low location, extending to the anal canal.

Of the five tumors surgically or pathologically staged as T4, three were understaged by WE-CT as T3. WE-CT failed to recognize a bladder wall invasion (n
Table 1. Contingency table comparing results of WE-CT with pathological findings ${ }^{a}$

\begin{tabular}{llrrr}
\hline & \multicolumn{2}{l}{ Pathology } & & \\
\cline { 2 - 5 } WE-CT & $<\mathrm{T} 3$ & $\geq \mathrm{T} 3$ & $\mathrm{~N}+$ & $\mathrm{N}-$ \\
\hline$<\mathrm{T} 3$ & 8 & 3 & & \\
$\geq \mathrm{T} 3$ & 3 & 26 & 11 & 4 \\
$\mathrm{~N}+$ & & & 2 & 20 \\
$\mathrm{~N}-$ & & & \\
\hline
\end{tabular}

${ }^{a}$ Concordant results are highlighted in boldface type; $<\mathrm{T} 3$ includes $\mathrm{T} 1$ or $\mathrm{T} 2 ; \geq \mathrm{T} 3$ includes $\mathrm{T} 3$ or $\mathrm{T} 4 ; \mathrm{N}+$, at least one peritumoral nodule $\geq 5 \mathrm{~mm}$

$=1)$, a perineal invasion $(\mathrm{n}=1)$, and invasion of the posterior wall of the vagina $(n=1)$. In three patients, WE-CT overstaged T3 tumors as T4 due to a presumed invasion of the bladder wall in two cases (one was an infected tumor) (Fig. 2) and of the right ovary in one case.

\section{WE-CT staging of lymph nodes}

Of the 37 cases pathologically examined, 13 had positive lymph nodes $(35 \%)$ and 24 were negative. None of the tumors confined to the bowel wall (T1 or T2) showed lymph node metastases. From a total of 37 metastatic nodes found on pathological examination, one had a diameter of $10 \mathrm{~mm}$ and two had a diameter of 8 $\mathrm{mm}$. All the others did not exceed $7 \mathrm{~mm}$.

On WE-CT and when reading A was used $(\mathrm{N}+$ with at least one peritumoral node $\geq 5 \mathrm{~mm}$ in diameter), nodes adjacent to the tumor were found in 15 patients (Fig. 3), but in four cases pathological examination was negative for local lymph node involvement. Using this criteria, WE-CT had a sensitivity of $84 \%$, specificity of $83 \%$, positive predictive value of $73 \%$, negative predictive value of $91 \%$, and an accuracy of $84 \%$ (confidence interval $=0.89-2.90)($ Table 1$)$. The number of metastatic nodes detected on WE-CT and histology was concordant in two cases. Of the true-positive cases, WECT showed more nodes in four cases and fewer in five. All the false-positive patients $(n=4)$ had numerous inflammatory or congestive nodes ranging from 6 to 22 on pathological examination. Of the two false-negative patients, histology showed seven nodes, two with metastases, both smaller than $5 \mathrm{~mm}$.

When reading $\mathrm{B}$ was applied, the same two falsenegative results obtained with reading A were found, but 12 false-positives cases were now registered. Using reading $\mathrm{B}$ to predict lymph node involvement on WECT resulted on a sensitivity of $85 \%$, specificity of $33 \%$, positive predictive value of $41 \%$, negative predictive value of $80 \%$, and an accuracy of $51 \%$. 


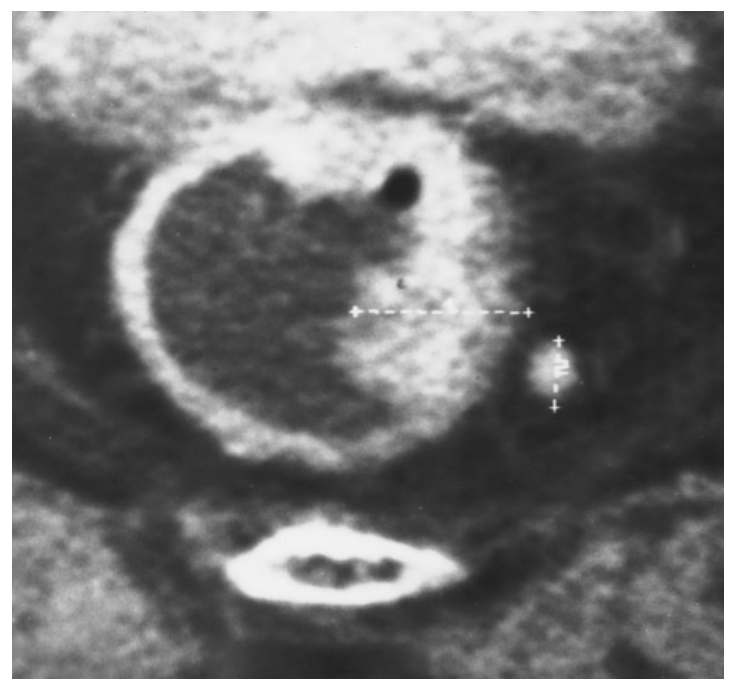

Fig. 3. T3 tumor showing a 7-mm nodule in the adjacent perirectal fat that corresponded, on pathological examination, to a metastatic lymph node.

\section{Comparison with TRUS}

TRUS was attempted in 25 patients. In seven (28\%), TRUS was either inconclusive or impossible to perform because the tumor was located beyond the limit of the rectal probe $(\mathrm{n}=5)$ or because of severe stenotic tumors $(\mathrm{n}=2)$. Data for comparison were available in 18 patients regarding the $\mathrm{T}$ stage and in 17 patients regarding the $\mathrm{N}$ stage (Fig. 4). On pathological examination, seven tumors were confined to the bowel wall (T1 or T2) and 11 were $\geq \mathrm{T} 3$. On TRUS, five patients were overstaged as $\mathrm{T} 3$, and three were understaged as $<\mathrm{T} 3$, for a sensitivity of $73 \%$, specificity of $29 \%$, positive predictive value of $62 \%$, negative predictive value of $40 \%$, and an accuracy of $39 \%$. On WE-CT, two cases were overstaged as T3, and one was understaged as $<\mathrm{T} 3$, for a sensitivity of $91 \%$, specificity of $71 \%$, and an accuracy of 83\% (Fig. 4A).

TRUS missed peritumoral nodal involvement in five patients, in contrast with WE-CT, where only one falsenegative result was found. TRUS showed a sensitivity of $29 \%$, specificity and positive predictive value of $100 \%$, a negative predictive value of $67 \%$, and an accuracy of $71 \%$, whereas WE-CT (when reading A was applied) showed values of $83 \%, 82 \%, 71 \%, 82 \%$, and $82 \%$, respectively (Fig. 4B).

\section{Discussion}

Previous experience using CT for the local staging of rectal tumors has been discouraging [9-11]. Compared with TRUS, CT is generally considered less accurate, but some of these CT studies were performed without
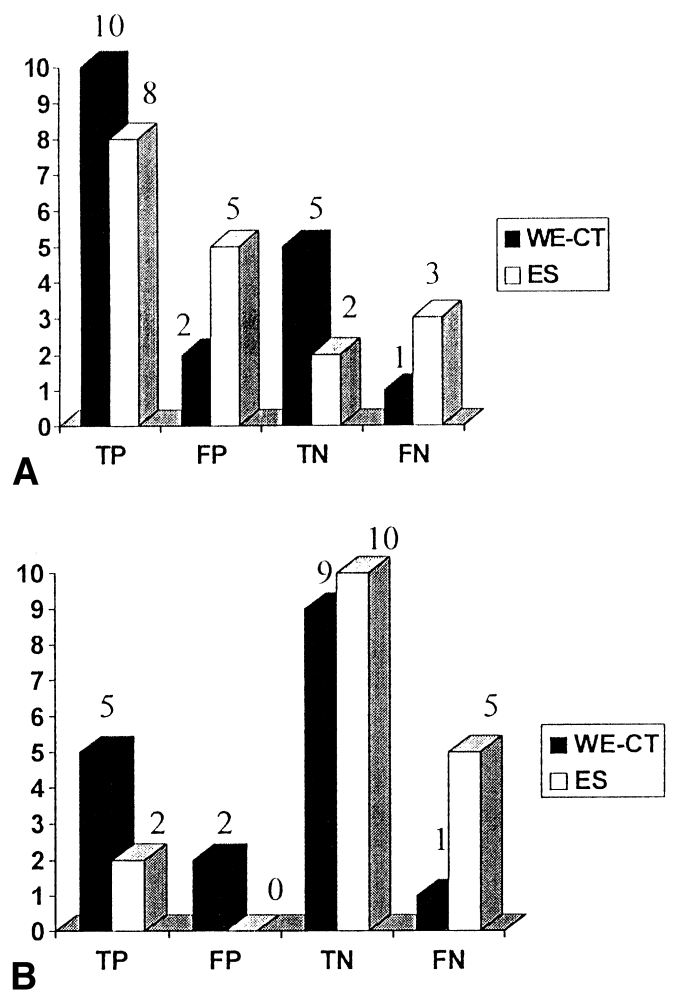

Fig. 4. Comparison between WE-CT and TRUS for T (A) and N (B) staging. TRUS showed a higher rate of false-positive and false-negative results for the $\mathrm{T}$ stage and false-negative results for the $\mathrm{N}$ stage. $\mathrm{TP}$, true positive; FP, false positive; TN, true negative; FN, false negative. For WE-CT, $\mathrm{N}$ was considered positive with at least one peritumoral node $\geq 5 \mathrm{~mm}$.

rectal distention $[13,14]$. The results we have obtained with WE-CT are in fact much better, approaching those reported in the literature when the same CT technique was applied [6]. This result means that when CT is performed with a technique of bowel distention, it can be a valuable tool for the local staging of low colorectal tumors. Rectal distention can be achieved by a variety of methods by using positive, negative, natural, or artificial contrast agents. The advantage of using water as a contrast agent, other than being widely available at practically no cost, is that it is less prone to cause artifacts and obviates the need of viewing images with different window settings for adequate reading [8]. WECT provided excellent results concerning detection and size estimation of the tumor, as also reported by Angelelli et al. [6] and Gazelle et al. [7] with a similar CT technique.

One of the major goals of preoperative staging of rectal neoplasms is to select patients who can benefit from adjuvant preoperative irradiation. Because suitable candidates for local radiotherapy are those presenting with deeply invasive and locally advanced lesions [1], it is important to identify those patients with transmural 
growth of the tumor or local metastatic adenopathy. In our study, the identification of the transmural growth of the tumor was more reliably accomplished by WE-CT, with TRUS showing a clear tendency to overstage (specificity of only 29\%). If patient management were based solely on the TRUS information, three patients would have been inappropriately selected for preoperative irradiation. This high rate of overstaging with TRUS is in agreement with the data provided by Hulsmann et al. [5] who reported a specificity of only $24 \%$ for TRUS. Inflammatory changes adjacent to the tumor are a well-known cause of $\mathrm{T}$ overstaging, for both CT and TRUS. However, results of the present comparative study showed that TRUS yielded a higher rate of falsepositive results without any obvious pathological explanation. Thus, this discrepancy may reflect the inherent subjectivity of TRUS versus that of WE-CT [15].

WE-CT had limitations. In the present study, it was not reliable for identifying the T4 stage. However, because T4 tumors were always staged by WE-CT as T3, patient management was not affected because, at our institution, tumor penetration of the bowell wall (T3 or T4 stage) is the main indication for preoperative irradiation. Results of WE-CT were less consistent with very low tumors, particularly when they extended to the anal canal. These results can be explained by the relative lack of fat planes in this anatomical area [16] and by the absence of significant distention by water enema.

Concerning detection of metastatic lymph nodes, WE-CT was superior to TRUS in showing good accuracy when the 5-mm diameter cutoff value was used. Angelelli et al. [6] reported an $88 \%$ sensitivity and $65 \%$ specificity for the detection of adenopathies, considering $\mathrm{N}$ positive any peritumoral nodule, irrespective of its size. Because we used thinner slices $(5 \mathrm{~mm})$, in many cases, we found tiny nodules in the adjacent fat that were responsible for the high rate of false-positive results when the criteria for reading B for metastatic lymph node involvement were applied. Thus, the use of a 5-mm lymph node diameter as a cutoff value seems to be a reasonable compromise when considering that the majority of metastatic nodes of rectal carcinoma are in fact very small [17].

Performance of WE-CT does not depend on tumor location, as TRUS does, or on the degree of stenosis, but with the recent use of flexible endoprobes, this problem may be overcome [18]. A real advantage of WECT is the possibility to image other lymphatic chains potentially involved in metastatic disease of rectal carcinoma such as the obturator, inguinal, or mesenteric nodes, which are not accessible to TRUS.

MRI with the use of transrectal coils may be a very promising technique for the local staging of rectal tumors, but, at present, costs are much higher and its real clinical impact is still to be proved $[19,20]$.
In conclusion, WE-CT is a safe and minor technical modification that allows an accurate local staging of low colorectal tumors. In our experience, WE-CT is more reliable than TRUS for the appropriate selection of patients for preoperative adjuvant therapy.

\section{References}

1. Fortier GA, Constable WC, Meyers H, et al. Preoperative radiation theraphy for rectal cancer: an effective therapy in need of a clinical trial. Arch Surg 1986;121:1380-1384

2. Tio TL, Coene PP, Delden OM, et al. Colorectal carcinoma: preoperative TNM classification with endosonography. Radiology 1991;179:165-170

3. Glaser F, Schlag P, Herfarth Ch. Transrectal ultrasonography for the assessment of invasion of rectal tumours and lymph node involvement. Br J Surg 1990;77:883-887

4. Orrow WJ, Wong WD, Rothenberger DA, et al. Transrectal ultrasound in the preoperative staging of rectal tumors. Dis Colon Rectum 1990;33:654-659

5. Hulsmans FJH, Tio TL, Fockens P, et al. Assessment of tumor infiltration depth in rectal cancer with transrectal ultrasonography: caution is necessary. Radiology 1994;190:715-720

6. Angelelli G, Mascarini L, Lupo L, et al. Rectal carcinoma: CT staging with water as contrast medium. Radiology 1990;177:511514

7. Gazelle GS, Gaa JA, Saini S, et al. Staging of colon carcinoma with water enema CT. J Comput Assist Tomogr 1995;19:87-91

8. Baert AL, Roex L, Wilms G, et al. Computed tomography of the rectum with water as contrast agent. Gastrointest Radiol 1989; $14: 345-348$

9. Adalsteisson B, Glimelius B, Graffman S, et al. Computed tomography in staging of rectal carcinoma. Acta Radiol Diagn 1985;26:45-55

10. Balthazar E, Megibow A, Hulnick A, et al. Carcinoma of the colon: detection and preoperative staging by CT. AJR 1988;150:301-306

11. Freeny P, Marks WM, Ryan JA, et al. Colorectal carcinoma evaluation with CT: preoperative staging and detection of postoperative recurrence. Radiology 1986;158:347-353

12. UICC. TNM classification of malignant tumors, 4th ed. P Hermaneck, LH Sobin, eds. New York: Springer, 1987

13. Rifkin MD, Ehrlich SM, Marks G. Staging of rectal carcinoma: prospective comparison of transrectal US and CT. Radiology 1989;170:319-322

14. Goldman S, Arvidsson H, Norming U, et al. Transrectal ultrasound and computed tomography in preoperative staging of lower rectal adenocarcinoma. Gastrointest Radiol 1991;16:259-263

15. Thoeni RF. Colorectal cancer: cross-sectional imaging for staging of primary tumor and detection of local recurrence. AJR 1991;156:909-915

16. Thoeni RF. CT evaluation of carcinomas of the colon and rectum. Radiol Clin North Am 1989;27:731-741

17. Dworák O. Number and size of lymph nodes metastases in rectal carcinomas. Surg Endosc 1989;3:96-99

18. Nielsen MB, Pedersen JF, Christiansen J. Rectal endosonography in the evaluation of stenotic rectal tumors. Dis Colon Rectum 1993;36:275-279

19. Chan TW, Kressel HY, Milestone B, et al.Rectal carcinoma: staging at MR Imaging with transrectal surface coil. Radiology 1991;181:461-467

20. Schnall MD, Furth EE, Rosato EF, et al. Rectal tumor stage; correlation of transrectal $\mathrm{MR}$ imaging and pathologic findings. $R a$ diology 1994;190:709-714. 